\title{
薯 Sonta
}

Desde la intervención

\section{Análisis de la intervención de Trabajo Social en una institución cultural en Hermosillo, Sonora, México}

\author{
Rosa María Islas Durón*, Graciela Ibarra López**
}

\begin{abstract}
Resumen
Este artículo repara sobre el quehacer del Trabajo Social dentro de una institución cultural, específicamente nos referimos a una investigación realizada en la "Casa de la Cultura de Hermosillo". El propósito fue analizar la intervención de Trabajo Social dentro de las Políticas Culturales, para ello describiremos dos programas que se desarrollan en ésta institución y las acciones que Trabajo Social realiza dentro del quehacer cultural: a) Función de Trabajo Social dentro de la Dirección Artística y b) Intervención del Trabajo Social en el programa de Promoción Cultural Comunitaria.
\end{abstract}

\begin{abstract}
This article is about the job of Social Work in a cultural institution, specifically we refer to the experience of our students in "Casa de la Cultura de Hermosillo". The purpose was analyzing the intervention of Social Work in the Cultural Politics, for this we will describe two cultural programs that develop in this cultural institution, and the actions that Social Work does inside the cultural tasks, this are: a) Social Work function in artistic direction Social Work Intervention in the Community Cultural Promoting program.
\end{abstract}

\section{Introducción}

Tradicionalmente el campo del Trabajo Social se ha orientado hacia diversas áreas fundamentales en el desarrollo de las comunidades como son: salud, educación, vivienda, trabajo etcétera. Este enfoque ha respondido a las necesidades sociales que la población demanda, que a su vez son objeto de intervención del Trabajo Social. Todos estos espacios han sido motivo de estudio por parte de profesionales de esta disciplina; pero existen otros factores que son producto de la vida moderna; necesidades que a pesar de estar latentes en la población no son manifestadas explícitamente, como es el caso de la cultura, un área poco explorada por este profesional. En Hermosillo, existen algunas instituciones con programas culturales, pero sólo la Casa de la Cultura de Hermosillo es la que cuenta con profesionistas de Trabajo social.

Es por ello que nos interesamos en profundizar e investigar la cultura como objeto de intervención del Trabajo Social, a partir de la investigación en este campo surgió la idea de elaborar un análisis de la intervención del Trabajo Social en estas instituciones, y lo abordaremos de la siguiente manera: Primeramente presentaremos un marco referencial de la Casa de la Cultura de Hermosillo, posteriormente un marco teórico de la cultura como objeto de intervención del Trabajo social, así como un análisis de la acción profesional del Trabajo social, y la caracterización de los usuarios, para finalizar con las conclusiones.

\section{Marco de referencia de la Casa de la Cultura de Hermosillo}

Inicia actividades por convenio de colaboración cultural que celebró el Estado libre y soberano de Sonora, representado por el entonces gobernador constitucional, Dr. Samuel Ocaña García, y el Instituto Nacional de Bellas Artes, representado por el director general, Lic. Juan José Bremer.

Según Sotomayor (1993) dicho acuerdo fue firmado en la Cd. de México el 2 de Mayo de 1980, apoyado en los artículos 4to y 24 de la Ley Orgánica del Poder Ejecutivo de Sonora, donde se crea la Casa de la Cultura del Estado de Sonora (hoy de Hermosillo), como una institución dependiente del Gobierno del Estado, a través de la Coordinación Estatal de Cultura y del Instituto Nacional de Bellas Artes. Su función quedó establecida para la enseñanza artística, investigación, difusión cultural, fomento de las artes, desarrollo de museos, bibliotecas, galerías, teatro, etcétera.

Podemos observar que desde el marco jurídico quedan establecidas las funciones de la Casa de la Cultura, entre las cuales se encuentra la investigación,

* Departamento de Trabajo Social. Universidad de Sonora. risla@sociales.uson.mx

** Departamento de Trabajo Social. Universidad de Sonora. gibarra@sociales.uson.mx 


\section{就 Sorvia}

por lo que esta institución no sólo debe atender actividades relacionadas con las Bellas artes, sino también debe contemplar el aspecto de la investigación, sobre todo aquellas relacionadas con las necesidades culturales, acciones que están directamente relacionadas con el Trabajo Social; ya que a través de las estrategias de acción de este departamento se pueden investigar las necesidades culturales de usuarios y no usuarios.

El día 10 de Octubre de 1980 fue una fecha trascendental para los sonorenses. Abre sus puertas en la región la primera institución con programas culturales, la Casa de la Cultura de Sonora, con el

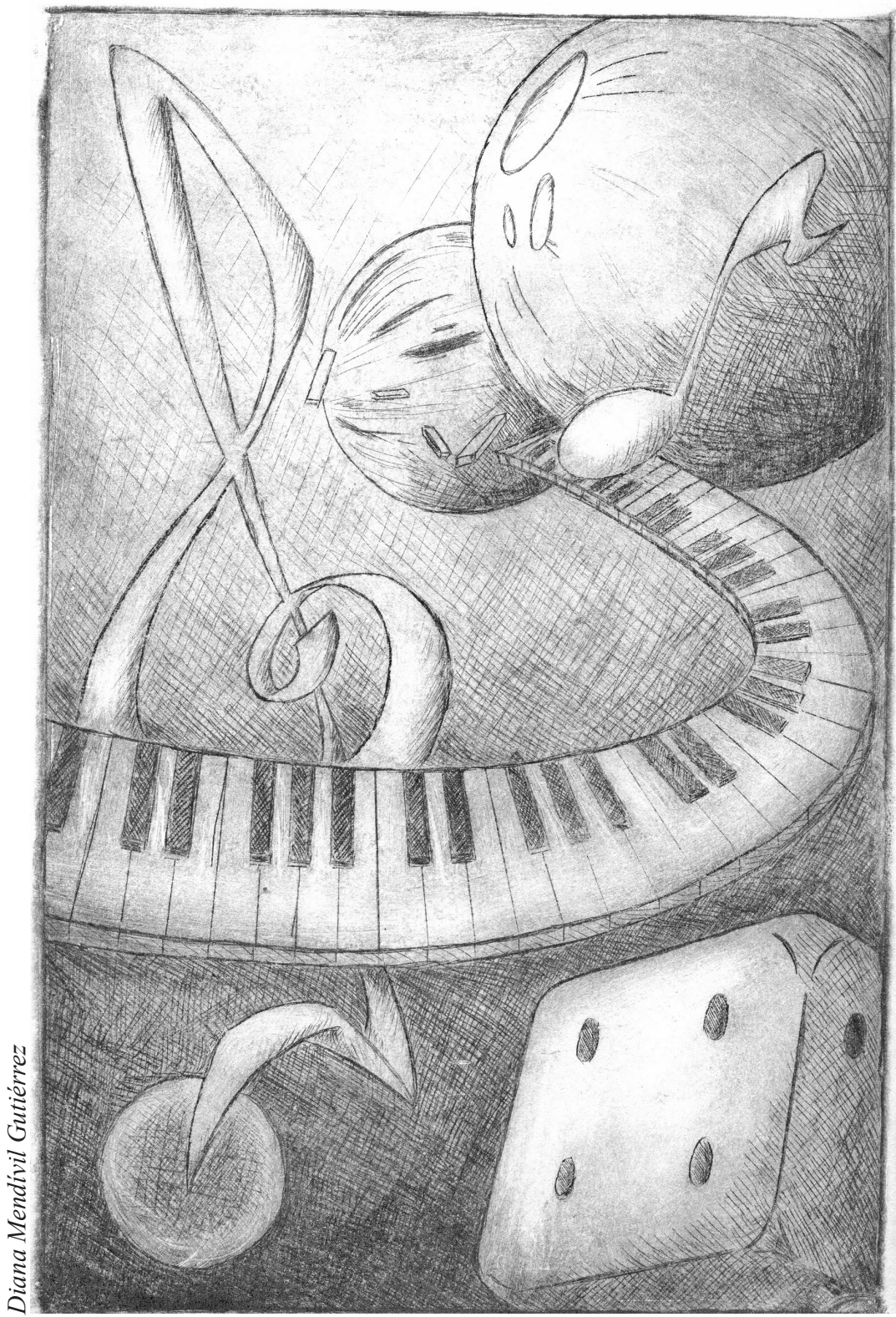

siguiente objetivo: "Promover, preservar, todas las manifestaciones culturales, municipales, estatales y cualesquier otra que reafirme nuestra identidad nacional" (Sotomayor, 1993,10).

En el objetivo general de la Casa de la Cultura observamos cómo se reflejan los fines de la Política Cultural de ese entonces, que giraban en torno al rescate, promoción y reafirmación de valores culturales, tanto municipales como estatales, con el fin de conservar una identidad nacional. Este objetivo en ningún momento menciona un desarrollo cultural integral del individuo o de capacitar a la persona en sus habilidades materiales y no materiales.

Para alcanzar este objetivo se crearon los siguientes departamentos: enseñanza artística, comunicación social, fomento y promoción de las artes, consejo consultivo, consejo pedagógico, sección de galerías, sección de radio, sección de biblioteca, sección de museos, sección de teatro. Con estos departamentos se puso en marcha la Casa de la Cultura de Sonora, áreas que se han ido incrementando al paso de tres décadas.

Cada departamento tiene sus respectivos programas, pero para nuestro análisis sólo nos enfocaremos en dos de ellos, que son los que cuentan con el profesional del Trabajo Social: Dirección de Educación Artística y el Programa de Promoción Cultural Comunitaria.

\section{Marco teórico, la cultura como objeto de intervención del Trabajo Social}

En Sonora el Trabajo Social se ha orientado tradicionalmente hacia diversas áreas fundamentales en el desarrollo de las comunidades, como son: salud, educación, vivienda, jurídico, penitenciario, trabajo, entre otros. Un campo que es objeto de estudio para el Trabajo Social, y que no ha sido explorado, es la cultura. Para conceptualizar la cultura es necesario que se tenga una relación directa con este concepto, así tenemos que existe una diversidad de definiciones, pero para el presente trabajo, que trata de ubicarla como objeto de intervención del Trabajo Social, 
presentaremos algunas definiciones que se insertan y tienen una relación más estrecha con el quehacer del Trabajo Social.

Marcuse, en su libro Ética de la Revolución (1969), cita a Webster, quien define la cultura como: "Complejo especifico de creencias, logros, tradiciones, etc., que forman el trasfondo de una sociedad, lenguaje, tradiciones, no llama logro a la destrucción, ni tradiciones a la crueldad y al fanatismo" (p.157). Webster circunscribe la cultura a un campo más selecto, es decir, al trasfondo de una sociedad matizada por aquellas actividades que constituyen su esencia y los separa en valores positivos, es decir que las finalidades que la sociedad misma tiene establecidas para la realización de las metas propuestas, y aquellas actitudes aisladas que no son aceptadas por los miembros que la integran, como actos delictivos, fanatismo derivado de una interpretación errónea de la religión. El autor señala como elementos determinantes de la cultura los valores propiamente dichos y excluye todo aquello que contribuye a la destrucción, es decir, actitudes que no responden al concepto puro de creencias, o bien que puedan ser calificadas de crueles y enajenantes. La definición que Webster nos presenta de cultura puede adaptarse a las instituciones culturales, sobre todo a los objetivos que éstas plantean, o bien puede reflejar las políticas culturales que el Estado planea en relación a la preservación de la cultura, rescate de las tradiciones y conservación de la identidad nacional. Por lo tanto el Trabajo Social, que es una profesión que interviene en la atención de las necesidades y trata de incidir en el bienestar social, puede relacionarse con este concepto, ya que estas tradiciones, creencias y valores pueden ser tratados a través de estrategias de acción del trabajo social, como son la investigación, promoción, difusión, etcétera.

Asimismo, en forma general, González (1981) define la cultura como un espectro que: "Abarca los valores ideológicos, aspiraciones y actitudes, modo de vida y costumbres, patrones de relaciones sociales comunes a un grupo fundamentadas, y en última instancia, condicionadas a las formas de producción especificas de un momento histórico determinado" (p. 68). Al definir la cultura, el autor anterior considera como aspectos importantes los valores, pero a diferencia de Webster, éste sitúa el concepto en un contexto más amplio, ya que no son sólo los valores ideológicos los que toma en cuenta, sino las aspiraciones, las actividades, las formas de vida. Elementos que fundamentan y condicionan las formas específicas de producción que se presentan en ciertos momentos históricos.

El estado implementa políticas culturales visualizadas desde su perspectiva como la conservación de los valores, en las que muchas instituciones culturales encuentran su razón de ser. Ahora bien, el Trabajo Social, al tomar como objeto de intervención a la cultura, dirige su acción a reconocer las actitudes, los valores ideológicos, formas de vida, y encaminarlas hacia la promoción y difusión de un proceso cultural que no sea circunstancial, o que no sólo atiendan aquellos aspectos que le atañen al Estado.

Otra manera de definir la cultura según Ortega (1992) es: "Toda producción humana que el hombre realiza en la vida social. Desde la fabricación de utensilios, hasta las grandes manifestaciones arquitectónicas, estáticas y estructuradas, complejas en el lenguaje" (p. 5). Para este autor, la cultura es todo lo que el hombre crea y transforma en la vida, desde el objeto más pequeño hasta las grandes manifestaciones arquitectónicas. Por lo tanto las instituciones culturales deben estar encaminadas, sobre todo, a realizar programas que llevan a cabo el reconocimiento de individuos, grupos o comunidades que sean capaces de crear y proyectar hacia la sociedad cultura.

En este sentido el Trabajo Social debe servir de enlace entre las instituciones y la población, a través de los programas que se llevan a las comunidades, así como investigar la creatividad de los individuos para reconocer lo que éstos son capaces de producir. En dicha detección o sondeo que se lleve a cabo, pueden descubrirse las capacidades materiales y espirituales que posee el hombre en las diferentes formas culturales, desde el arte culinario hasta los creadores de las bellas artes, entendiendo por éstas la música, pintura, teatro, poesía, etcétera.

Por otro lado al convertirse la cultura en un objeto de intervención del Trabajo Social, no podemos dejar de lado las políticas culturales, ya que en nuestro Estado todas las instituciones culturales, como también los programas, se desprenden de éstas. Por ello consideramos necesario definirlas. García Canclini (1987), define: "Entendemos por Políticas Culturales el conjunto de intervenciones realizadas por el Estado, las instituciones civiles, y los grupos comunitarios, organizados a fin de orientar el desarrollo simbólico, satisfacer las necesidades culturales de la población y obtener consenso para un tipo de orden de 


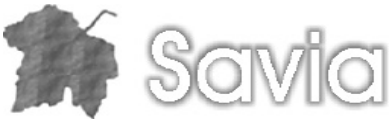

transformación social"' (p. 28). La manera como el autor define la política cultural es bastante amplia, ya que para él no sólo son las formas que implementa el Estado, sino también toma en cuenta todas aquellas acciones culturales que grupos dentro de la sociedad realizan, descartando el papel que juega la iglesia en este aspecto.

\section{Análisis de la acción profesional del Trabajo Social}

Se cuestionó a las entrevistadas sobre cuáles actividades llevaban a cabo dentro de las funciones inherentes al trabajo social como: Investigación, planeación, evaluación, educación, promoción, administración y gestión, obteniéndose las siguientes conclusiones: en lo referente a las actividades de investigación, opinaron que no las realizan, sin embargo desarrollan ciertos procesos de tipo preliminar, sin llegar a detectar realmente las necesidades culturales sentidas por la población. Con respecto a la planeación, las entrevistadas sólo tienen una leve idea de lo que es la planeación, entendiéndose ésta como el conjunto de procesos y actividades que ayudan a organizar los recursos existentes para el logro de objetivos y metas en la atención de problemas sociales. Con base a lo anterior se puede decir que las entrevistadas sólo tienen una leve idea de lo que es la planeación, ya que en sus respuestas mencionan la identificación de recursos como parte de la misma sin profundizar en ello.

También se advirtió que no realizan una evaluación como parte de su intervención, lo que limita su desarrollo profesional. Sólo realizan evaluación de las actividades desarrolladas dentro del departamento donde están ubicadas y no trasciende a nivel institucional.

Si consideramos función educativa lo que Ander Egg (1995) define como: "Un proceso que implica acciones sistémicas y metódicas del Trabajador Social, sobre el usuario con el fin de favorecer el desarrollo de cualidades morales, intelectuales o físicas de los individuos" (p.103). Podemos concluir que lo que las entrevistadas consideran como proceso educativo el atender y proporcionar servicios a los alumnos. Acciones que no cumplen procesos educativos en sí, sino más bien son labores que están encaminados a proporcionar servicios esporádicos a los usuarios que lo solicitan, y no llevan un proceso como tal, dentro de la institución.

Respecto a la función de promoción, consideramos que son las adecuadas, ya que utilizan diferentes técnicas de comunicación, como volantes, carteles, medios de comunicación, visitas domiciliarias, radio, prensa, televisión, visitas institucionales a centros educativos, periódico mural, entre otras. Con relación a la función de administración y gestión se puede apreciar que sí realizan esta actividad, ya que dentro de sus funciones

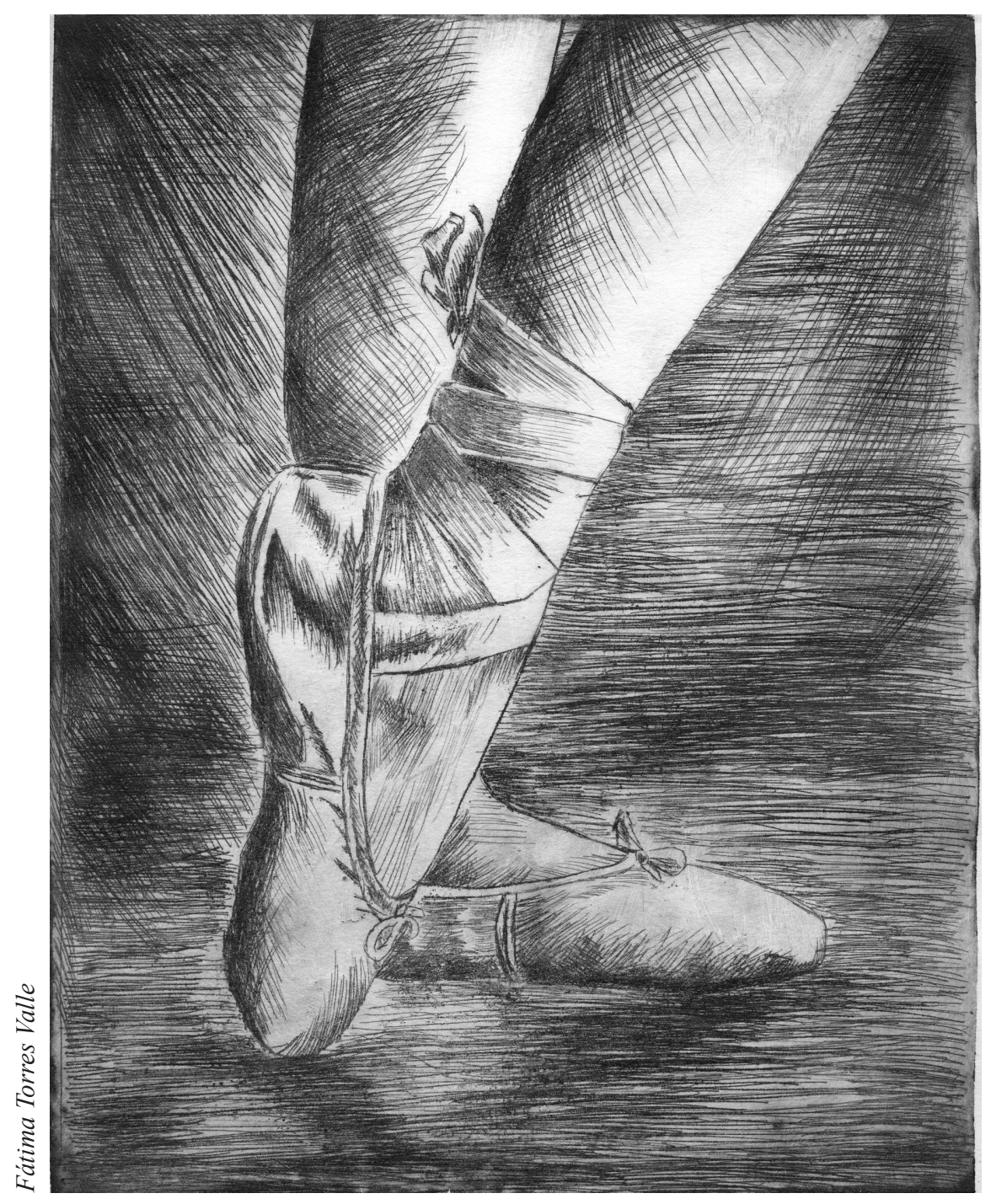


está la de coordinarse con otras instituciones para la impartición de pláticas, talleres, eventos artísticos y culturales.

Se interrogó a las trabajadoras sociales de la Casa de la Cultura sobre si realizan otras funciones, además de las ya mencionadas. Algunas respuestas fueron que promover la cultura mexicana y las aptitudes culturales de las personas con las que trabajan. Por lo anterior podemos deducir que estas últimas funciones que mencionaron, y que consideran relacionadas con las antes cuestionadas, no son propias del Trabajo Social, ya que la función principal que predomina en su acción profesional varía de acuerdo al objetivo de cada uno de estos departamentos donde están ubicadas.

\section{Conclusiones}

- La aproximación teórica nos permite visualizar a la cultura como objeto de intervención del Trabajo Social.

- Las Instituciones culturales son instancias que pueden ser intervenidas por el Trabajo Social, donde se actuaría como intermediario entre las

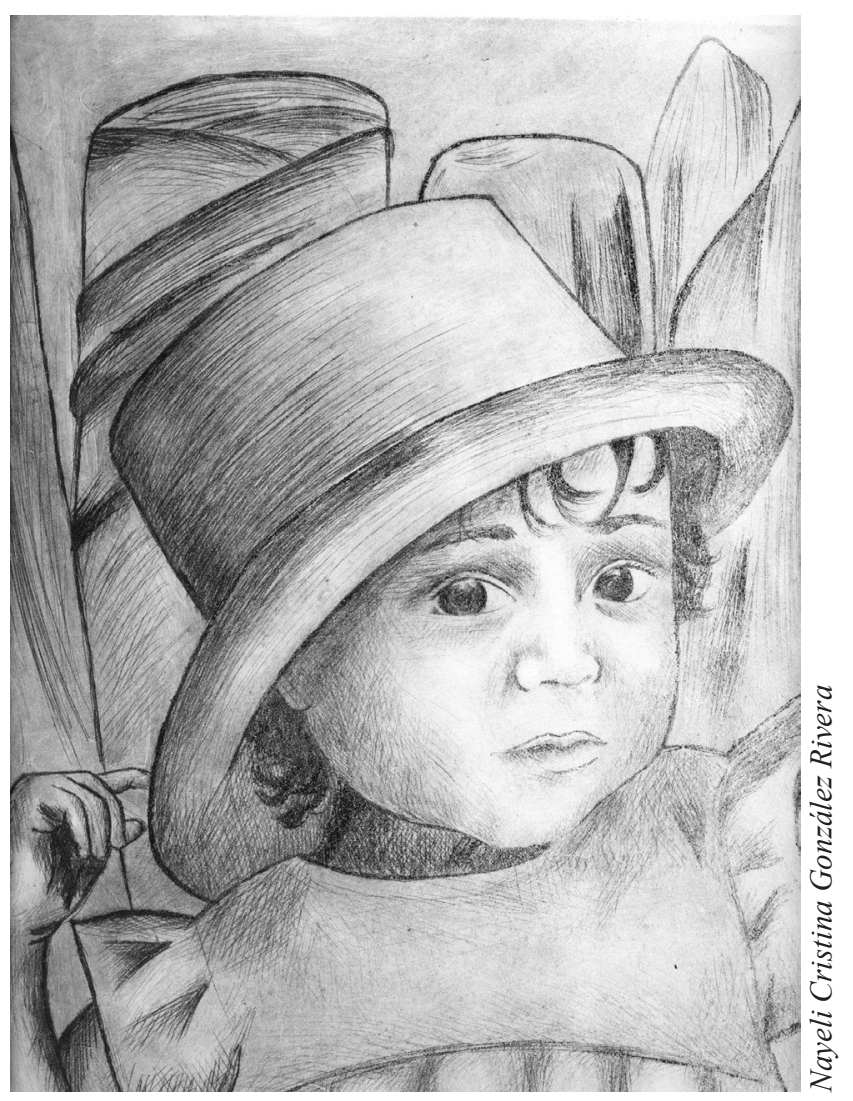

necesidades culturales de la población y las políticas culturales.

- La acción profesional del Trabajo Social es muy limitada, por la poca participación que les permiten los supervisores.

- Hace falta investigación por parte del profesional del Trabajo Social para detectar las demandas culturales de la población, así como de la aceptación de los programas.

- Los profesionales del Trabajo Social de la Casa de la Cultura no están desarrollando una función intermediaria entre recursos y demandas de la población en materia de Programas Culturales, sólo se enfocan a actividades administrativas.

- La intervención del Trabajador Social debe incrementarse dentro de las instituciones culturales.

- Es importante señalar que la cultura es un área potencial para la intervención del profesional del trabajo social, sin embargo las funciones de éstos son poco valoradas.

\section{Bibliografía}

Ander-Egg, E. (1995). Diccionario de Trabajo Social. Buenos Aires, Argentina: Editorial Ateneo.

Celaya, L. (1990). Programa de Promoción Cultural Comunitaria de la Casa de la Cultura de Hermosillo. Hermosillo, Sonora, México.

García Canclini, N. (edit.) (1987). Políticas Culturales en América Latina. México: Editorial Grijalbo.

González, R. (1981). La Frontera del Norte. México: Editorial Colegio de México.

Guillén, M. y Terán, G. G. (1990). Las prácticas de Reconocimiento de la Licenciatura en Trabajo Social, Una Propuesta Preliminar. México: Editorial UNISON.

Marcuse, H. (1969). Ética de la Revolución. España: Editorial Cal, Ensayistas de hoy.

Memoria. Casa de la Cultura de Hermosillo 19801990. Hermosillo, Sonora: Casa de la Cultura de Hermosillo.

Ortega, M. (1992). Revista Chasqui, Revista Latinoamericana de Comunicación, No. 12

Sotomayor, M. (1993). Propuesta de Trabajo y Compromiso para la Casa de la Cultura de Hermosillo. 